\title{
Assessing Adherence to Antihypertensive Therapy in Primary Health Care in Namibia: Findings and Implications
}

\author{
M. M. Nashilongo ${ }^{1}$ B. Singu ${ }^{1} \cdot$ F. Kalemeera ${ }^{1} \cdot{\text { M. } \text { Mubita }^{1} \cdot \text { E. Naikaku }}^{1} \cdot$ A. Baker $^{2}$.

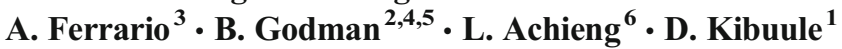

Published online: 14 October 2017

(C) The Author(s) 2017. This article is an open access publication

\begin{abstract}
Introduction Namibia has the highest burden and incidence of hypertension in sub-Sahara Africa. Though non-adherence to antihypertensive therapy is an important cardiovascular risk factor, little is known about potential ways to improve adherence in Namibia following universal access. The objective of this study is to validate the Hill-Bone compliance scale and determine the level and predictors of adherence to antihypertensive treatment in primary health care settings in sub-urban townships of Windhoek, Namibia.

Methods Reliability was determined by Cronbach's alpha. Principal component analysis (PCA) was used to assess construct validity.

Results The PCA was consistent with the three constructs for 12 items, explaining 24.1, 16.7 and $10.8 \%$ of the
\end{abstract}

variance. Cronbach's alpha was 0.695 . None of the 120 patients had perfect adherence to antihypertensive therapy, and less than half had acceptable levels of adherence $(\geq 80 \%)$. The mean adherence level was $76.7 \pm 8.1 \%$. Three quarters of patients ever missed their scheduled clinic appointment. Having a family support system $(\mathrm{OR}=5.4,95 \%$ CI 1.687-27.6, $p=0.045)$ and attendance of follow-up visits $(\mathrm{OR}=3.1,95 \%$ CI $1.1-8.7, p=0.03)$ were significant predictors of adherence. Having HIV/ AIDs did not lower adherence.

Conclusions The modified Namibian version of the HillBone scale is reliable and valid for assessing adherence to antihypertensives in Namibia. There is sub-optimal adherence to antihypertensive therapy among primary health cares in Namibia. This needs standardized systems to strengthen

M. M. Nashilongo

magdaleenanash@gmail.com

B. Singu

bsingu@unam.na

F. Kalemeera

fkalemeera@unam.na

M. Mubita

mmubita@unam.na

E. Naikaku

enaikaku@unam.na

A. Baker

amanj.baker@strath.ac.uk

A. Ferrario

A.Ferrario@1se.ac.uk

\author{
L. Achieng \\ loicea.la@gmail.com \\ D. Kibuule \\ dkibuule@unam.na
}

1 School of Pharmacy, Faculty of Health Sciences, University of Namibia, Box 13301, 340 Mandume Ndemufayo Avenue Pioneers Park, Windhoek, Namibia

2 Strathclyde Institute of Pharmacy and Biomedical Sciences, University of Strathclyde, Glasgow, UK

3 LSE Health, London School of Economics and Political Science, London, UK

4 Department of Laboratory Medicine, Division of Clinical Pharmacology, Karolinska Institute, Karolinska University Hospital Huddinge, Stockholm, Sweden

5 Health Economics Centre, Liverpool University Management School, Liverpool, UK

6 Department of Medicine, University of Nairobi, Nairobi, Kenya 
adherence monitoring as well as investigation of other factors including transport to take full advantage of universal access.

Keywords Adherence $\cdot$ Hypertension $\cdot$ Namibia $\cdot$ Primary health care $\cdot$ Universal access

\section{Introduction}

Cardiovascular diseases (CVD) remain a significant health problem in lower and middle income countries (LMICs) including Namibia [1, 2]. In 2001, three out of four patients or more with hypertension lived in LMICs particularly in the Africa region [3-5]. In 2008, an estimated 17 million people died from CVD globally $[1,6,7]$. In the same year, more than half of CVD-related deaths (9.4 million) were due to hypertension [6]. The majority were premature due to uncontrolled blood pressure [8].

In Namibia, CVD accounted for $21 \%$ of annual deaths in 2012 [9], with the prevalence of hypertension among adults aged between 35 and 64 at between 44 and $45 \%[9,10]$, appreciably higher than the pooled prevalence rates of $30 \%$ in sub-Sahara Africa [2]. However, among patients with hypertension in LMICs, only between 33 and $66 \%$ of them are currently receiving antihypertensive medicines [4]. This prevalence and mortality level demands strengthening and scaleup of health care systems, including primary health care facilities in LMICs, to prevent, manage, and control hypertension, to improve health outcomes in the future $[1,6,11]$. As a result, it helps achieve sustainable development goal (SDG) 3.4, aiming to reduce premature mortality from noncommunicable diseases (NCDs) by one third from current levels by 2030 [12]. This includes strategies to optimize adherence to antihypertensive therapy $[6,13,14]$, although this may not always be the case [15], as well as enhance access to affordable medicines to treat NCDs including hypertension by $80 \%$ [12].

To address this considerable and growing public health problem, primary health care ( $\mathrm{PHC}$ ) centres and policies in Namibia now provide for universal access to essential antihypertensive medicines as well as other aspects of care at no cost [16-19]. PHC facilities in Namibia are strategically located among under privileged communities and play a critical role in the access to care for patients with hypertension. Universal access reduces a financial barrier to accessing antihypertensive medicines, which can be a concern in LMIC with high copayment levels $[2,20]$. However, this raises the question on the extent of other factors involved in subsequent poor levels of adherence to antihypertensive medicines in LMICs if this still occurs following universal access.

Consequently, the aim of this study is to determine the levels and predictors of compliance to antihypertensive medicines among patients receiving care at PHC facilities in four sub-urban townships in the capital city, Windhoek. In addition, this study also aims to validate the Hill-Bone compliance scale. The findings will be used to suggest future policies in Namibia and wider to improve the management of hypertensive patients.

\section{Methods}

\section{Study Design}

A descriptive cross-sectional observational study was undertaken. The study included patients initiated on antihypertensive medication at public PHCs in four sub-urban townships of Windhoek. There are a total of seven PHCs in Windhoek; however, only four are located in peri-urban settings similar to other situations in sub-Sahara regions. Those in the central business district of Windhoek were excluded due to their cosmopolitan patient population. The chosen PHCs are based in Okuryangava, Otjomuise, Donkerhoek and Hakahana. These four PHCs are the only public outpatient clinics in these townships that provide primary health care services, and this is mainly to low socioeconomic groups, the majority of whom do not have medical insurance cover [21]. In Namibia, antihypertensive therapy is initiated at hospital level, with patients subsequently accessing free follow-up care and medication refills at PHCs and other centers.

The main outcome measure was the proportion of patients with adherence levels to antihypertensive therapy $\geq 80 \%$ on the Hill-Bone blood pressure scale, in line with previous publications $[6,22]$. The secondary outcome measure was predictors of adherence to antihypertensive medication.

Patients were selected using a systematic sampling method based on the daily attendance registers. The target sample was 30 patients per $\mathrm{PHC}$ facility as recommended by the $\mathrm{WHO} /$ INRUD method for measuring medicine use in the community [23]. The study included all patients who had confirmed diagnosis of hypertension, had completed at least one cycle (6 months) of antihypertensive medication refill at the PHC, were aged more than 18 years, were able to recognize and tell apart their antihypertensive medicines from any other daily medicines and had given written informed consent to participate in the study.

Out of a total of 185 patients selected, 143 patients met the eligibility criteria, with 42 patients not routinely (> 6 months) receiving antihypertensive refills at the four PHCs under investigation. The study excluded 23 more patients. Seven did not consent to participate, four were too ill to participate in the interviews, three had incomplete or incoherent records regarding their clinical characteristics and antihypertensive therapies in their health records (passports) and a further nine were due to systematic selection of patients to meet the sample size (Fig. 1). 
Fig. 1 Flow chart for patient sample selection

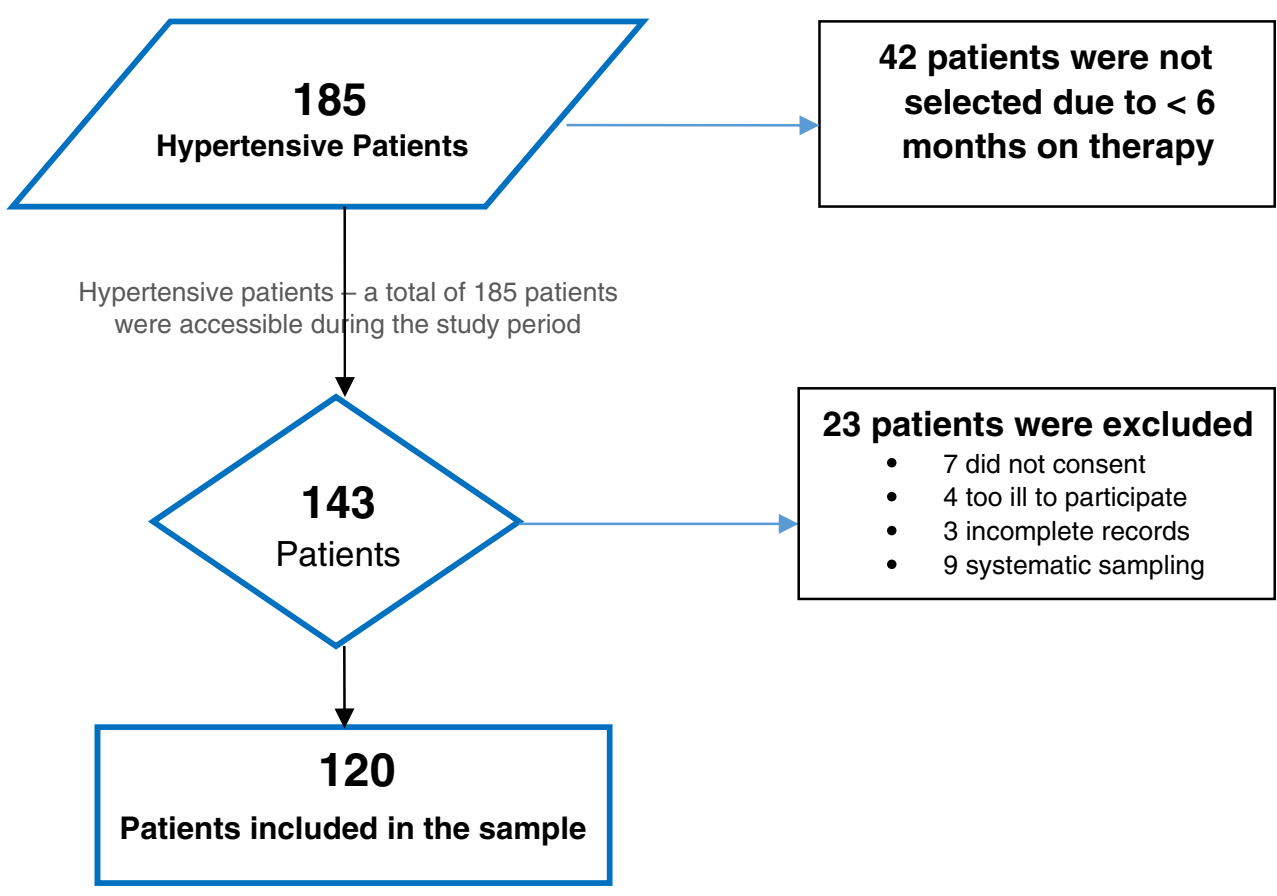

\section{Validation of the Hill-Bone Scale and Data Collection}

The reliability of the Namibian version of the Hill-Bone scale was determined by the Cronbach's alpha. Principal component analysis (PCA) was used to assess construct validity. The questionnaire, after taking details of the patient characteristics and their medical history (Sections A and B), was structured according to the Hill-Bone compliance to high blood pressure therapy scale (HBCHTS) [24, 25]. The scale assesses patient behaviours for three important behavioural domains of high blood pressure treatment, which are reducing sodium intake, appointment keeping and medication taking [24]. The HBCHTS has been validated in a number of countries including among black Africans in South Africa, a country with similar primary health care centres and cultures to Namibia [25-28]. The HBCHTS scale was preferred over the Morisky Medication Adherence Scale (MMAS) methods in this study due to the variety of items used to assess adherence. This is because the HBCHTS questionnaire is 'based on the Morisky scale and is specific to antihypertensive therapy and assesses items pertaining to lifestyle modification in the setting of hypertension' [24].

\section{Data Collection}

The data was collected from 11 July until 3 August 2016 by the researchers led by SN, MM and DK. On the day of data collection, every other second patient on the daily attendance register was interviewed using a pre-tested structured questionnaire (Appendix A). The first question before the formal questions started was whether the patient was currently taking any medication for hypertension. If the answer was no, the interview was stopped and the patient was excluded from the study. Interviews were conducted in Afrikaans and in Oshiwambo, the most commonly spoken local languages in Namibia.

The interview question items of the HBCHTS were adapted to Namibia's situation and used to test for the level of adherence to antihypertensive medication [24, 26]. The level and predictors of adherence to antihypertensive medication were analysed using quantitative analysis in SPSS software version 22 .

The HBCHTS scale has 14 question items with a 4-point Likert response format around four response categories to each question. These were the following: all the time-1 point, most of time -2 points, sometimes -3 points and none of the times - 4 points. Consequently, a maximum of 4 points where 4 indicates the poorest compliance and 1 indicates the best compliance. The scores were adjusted by the percentage of patients in each category to derive the mean and standard deviation for each of the 14 items (maximum of 4). The lower the mean score, the better the compliance with medication, appointment keeping and avoidance of salty and fast foods. Consequently, replies to questions with a mean score $>2$ were indicative of poor compliance.

The item and factor analyses showed reliability for the final 12 items chosen (Table 2). After adding the points of all 12 items of the modified scale, the total score should range from 12 to 48 points. Perfect adherence is equivalent to 12 points on the HBCHTS scale. The percentage adherence level per patient was subsequently calculated from the HBCHTS scores using the formula \%adherence $=\frac{(48-\text { Hill-Bone score }) \times 100}{36}$. The denominator (36) is the range of HBCHTS scores $(48-12)$. Adherence to antihypertensive therapy was categorized as 
perfect $(100 \%)$, acceptable $\geq 80 \%$, and non-adherence if less than $80 \%$ in line with previous studies $[6,22,29,30]$. Chisquare test was used to assess for associations between adherence and individual's characteristics. A multivariate logistic regression analysis was performed to adjust for confounders, and the results were presented as odds ratio (OR) and 95\% confidence interval.

\section{Ethics}

The study was approved by the research and ethics committee of the Faculty of Health Sciences, UNAM and the Ministry of Health and Social Services (MoHSS). All patients who participated in the study gave written informed consent. The management of the four PHCs, i.e. Okuryangava HC, Otjomuise, Donkerhoek and Hakahana, approved the study. In order to maintain confidentiality, questionnaires were coded and no patient specific identifiers such as names and hospital numbers were collected.

\section{Results}

\section{Bivariate Analysis of Sociodemographic and Clinical Characteristics}

A total of 120 patients with hypertension (i.e. responded 'yes' to the first question-Appendix A) completed the study (100\% response rate); the mean age was $47.3 \pm 11.1$ years. The majority of the respondents were female, attained at least primary level education, were unemployed and not married (Table 1). Approximately four out of every ten patients had at least one other chronic comorbidity in addition to hypertension, the most common being HIV/AIDS $(n=31-25.8 \%)$.

Most patients had adequate supplies of medicines to last them until the next PHC visit (Table 1). Three quarters of the patients $(75 \%)$ have ever missed their scheduled clinic appointment (Table 1). The main reasons were being work commitments $(n=54,60 \%)$ and/or feeling ill $(n=12,13 \%)$, forgetting the appointment day $(n=11$, $12 \%)$ or lacking of transport to the health facility $(n=14$, $15 \%)$. The majority of patients $(n=100,83.3 \%)$ reported receiving adequate information from health care providers on how to take their medication. However, over half of the patients were not knowledgeable of the consequences of hypertension and non-adherence to medication (Table 1). Most patients ( $n=94,78.3 \%)$ received support from friends and/or relatives in adhering to treatment and attending clinic appointments (the form of support included reminders to attend clinic appointments and to take medicines at the prescribed time).

\section{Reliability and Validity of the Namibian Version of the Hill-Bone Compliance Scale}

The reliability and construct validity of the Namibian HillBone compliance scale was determined using item and factor analysis. A modified scale consisting of 12 items (after exclusion of items 6 and 12 from the original scale) demonstrated acceptable internal consistency with a standardized Cronbach $\alpha$ of 0.70 (0.695), and item-total correlations all (>0.31), with mean inter-item correlation of 0.16 . The initial principal component analysis with a yielded four constructs of the Namibian version of the Hill-Bone compliance scale. A forced three structures with a KMO of 0.634 and a significant Bartlett's test of sphericity $(p<0.001)$ was consistent with the three constructs of the Hill-Bone scale with Eigen values and variances of $2.9(24.1 \%)$ for medication taking, 2.0 (16.7\%) for salt taking and 1.3 (10.8\%) for appointment keeping (Table 2).

\section{Compliance to High Blood Pressure Therapy According to the Hill-Bone Compliance Scale}

The mean HBCHTS score for the 12 items on the 4-Likert point scale was $1.7 \pm 0.2$, this ranged from 1.01 to $2.3(57.5 \%)$ by question item (Table 2). Most question items (10/12, $71.4 \%$ ) had a mean score $<2$. Only one item had a perfect mean score of 1 , which was ever missing taking medication because of carelessness. The three items that scored poorly (mean score $>2$ ) included items 3, 4 and 5 that assess the domain of salt intake and eating salty foods (2.2/4), using spices and additional salt on food (2.3/4) and eating fast food (2.1/4) (Table 2). Overall, no patients had perfect HBCHTS total score of 12. The mean HBCHTS score for the 12-item modified scale among the patients was $20.4 \pm 2.9$ out of a possible 48 points, with the IQR of 18-23 points.

According to the results of the bivariate analysis (Table 1), the following factors were significantly associated with good adherence to antihypertensive therapy (adherence $\geq 80 \%$ ): (i) attendance to follow-up visits ( $p=0.008$ ), (ii) a patient's age of less than 40 years $(p=0.025)$, (iii) having a treatment support buddy ( $p=0.024)$ and (iv) never having missed a clinic appointment $(p<0.001)$. There was a negative correlation between patients' ages and adherence to antihypertensive medication (Pearson's $r=-1.28, p=0.163$ ).

There was no association between patients' sociodemographic characteristics such as sex, employment status, education level and adherence to antihypertensive medication $(p>0.05)$ in the bivariate analysis (Table 1). There was also no association between adherence and any chronic co-morbidity and between adherence and HIV/AIDS specifically.

The mean adherence level was $76.7 \pm 8.1 \%$ with an IQR of 69.4-83.3\% (Fig. 2). None of the patients had perfect adherence, and less than half of the patients - $42.5 \%$ (50/120) - had acceptable levels of adherence to antihypertensive therapy, that is $\geq 80 \%$. 
Table 1 Bivariate analysis of characteristics of respondents and adherence $(n=120)$

\begin{tabular}{|c|c|c|c|c|c|}
\hline \multirow{2}{*}{$\begin{array}{l}\text { Characteristic } \\
\text { Total }\end{array}$} & \multirow{2}{*}{$\begin{array}{l}\text { Total }(\%) \\
120\end{array}$} & \multicolumn{2}{|c|}{ Adherence level } & \multirow[t]{2}{*}{ Crude OR (95\% CI) } & \multirow{2}{*}{$\begin{array}{l}p \text { value } \\
0.201\end{array}$} \\
\hline & & $\begin{array}{l}\geq 80(\%) \\
51(42.5)\end{array}$ & $\begin{array}{l}<80(\%) \\
69(57.5)\end{array}$ & & \\
\hline \multicolumn{6}{|c|}{ Distance of PHC from state hospital } \\
\hline$<3 \mathrm{~km}$ & $60(50)$ & $21(35)$ & $39(65)$ & $0.5(0.3,1.1)$ & 0.097 \\
\hline$>3 \mathrm{~km}$ & $60(50)$ & $30(50)$ & $30(50)$ & 1 & \\
\hline \multicolumn{6}{|l|}{ PHC facility } \\
\hline Okuryangava & $30(25)$ & $8(26.7)$ & $22(73.3)$ & $2.1(0.7,6.2)$ & 0.229 \\
\hline Otjomuise & $30(25)$ & $15(50)$ & $15(50)$ & $0.8(0.3,2.1)$ & 0.179 \\
\hline Hakahana & $30(25)$ & $15(50)$ & $15(50)$ & $0.8(0.3,2.1)$ & 0.605 \\
\hline Donkerhoek & $30(25)$ & $13(43.3)$ & $17(56.7)$ & 1 & 0.605 \\
\hline \multicolumn{6}{|l|}{ Age categories (years) } \\
\hline$<40$ years & $30(33.3)$ & $18(60.0)$ & $12(40.0)$ & $2.6(1.1,6.0)$ & $0.025 *$ \\
\hline$>40$ years & $90(66.7)$ & $33(36.7)$ & $57(63.3)$ & 1 & \\
\hline \multicolumn{6}{|l|}{ Patient's sex } \\
\hline Male & $51(42.5)$ & $20(39.2)$ & $31(60.8)$ & $0.8(0.4,1.6)$ & 0.532 \\
\hline Female & $69(57.5)$ & $31(44.9)$ & $38(55.1)$ & 1 & \\
\hline \multicolumn{6}{|l|}{ Employment status } \\
\hline Employed & $53(44.2)$ & $20(37.7)$ & $33(62.3)$ & $0.7(0.3,1.5)$ & 0.348 \\
\hline Unemployed & $67(55.8)$ & $31(46.3)$ & $36(53.7)$ & 1 & \\
\hline \multicolumn{6}{|l|}{ Education level } \\
\hline At least primary & $94(78.3)$ & $41(43.6)$ & $53(56.4)$ & $1.2(0.5,3.0)$ & 0.638 \\
\hline No education & $26(21.7)$ & $10(38.5)$ & $16(61.5)$ & 1 & \\
\hline \multicolumn{6}{|l|}{ Marital status } \\
\hline Single & $69(57.5)$ & $27(39.1)$ & $42(60.9)$ & $0.7(0.3,1.5)$ & 0.385 \\
\hline Espoused & $51(41.5)$ & $24(47.1)$ & $27(52.9)$ & 1 & \\
\hline \multicolumn{6}{|l|}{ Years with hypertension } \\
\hline$<10$ years & $87(72.5)$ & $39(44.8)$ & $48(55.2)$ & $1.4(0.6,3.2)$ & 0.402 \\
\hline$>10$ years & $33(27.5)$ & $12(36.4)$ & $21(63.6)$ & 1 & \\
\hline \multicolumn{6}{|l|}{ Chronic co-morbidity } \\
\hline Yes & $49(40.8)$ & $19(38.8)$ & $30(61.2)$ & $0.8(0.4,1.6)$ & 0.493 \\
\hline No & $71(59.2)$ & $32(45.1)$ & $39(54.9)$ & & \\
\hline \multicolumn{6}{|l|}{ HIV infection } \\
\hline Yes & $31(25.8)$ & $12(38.79)$ & $19(61.3)$ & $0.8(0.4,1.9)$ & 0.620 \\
\hline No & $89(74.2)$ & $39(43.8)$ & $50(56.2)$ & 1 & \\
\hline \multicolumn{6}{|c|}{ Has a treatment support buddy } \\
\hline Yes & $94(78.3)$ & $45(47.9)$ & $49(52.1)$ & $3.1(1.1,8.3)$ & $0.024 *$ \\
\hline No & $26(21.7)$ & $6(23.1)$ & $20(76.9)$ & 1 & \\
\hline \multicolumn{6}{|c|}{ Support buddy on clinic visits } \\
\hline Yes & $94(78.3)$ & 37 (44.6) & $46(55.4)$ & $1.3(0.6,2.9)$ & 0.490 \\
\hline No & $26(21.7)$ & $14(37.8)$ & $23(62.2)$ & 1 & \\
\hline \multicolumn{6}{|c|}{ Sufficient medication refills on last visit } \\
\hline Yes & $101(84)$ & $43(42.6)$ & $58(57.4)$ & $1.0(0.4,2.8)$ & 0.970 \\
\hline No & $19(16)$ & $8(42.1)$ & $11(57.9)$ & 1 & \\
\hline \multicolumn{6}{|c|}{ Informed about medicine } \\
\hline Well informed & $100(83)$ & $42(42)$ & $58(58)$ & $0.9(0.3,2.3)$ & 0.804 \\
\hline Not informed & $20(17)$ & $9(45)$ & $11(55)$ & 1 & \\
\hline Knows complications & & & & & \\
\hline Knowledgeable & $49(40.8)$ & $19(38.8)$ & $30(61.2)$ & $0.8(0.4,1.6)$ & 0.493 \\
\hline Not knowledgeable & $71(59.2)$ & $32(45.1)$ & $39(54.9)$ & 1 & \\
\hline Attend follow-up visits & & & & & \\
\hline Always & $30(25)$ & $19(63.3)$ & $11(36.7)$ & $3.1(1.3,7.4)$ & $0.008 *$ \\
\hline Sometimes & $90(75)$ & $32(35.6)$ & $58(64.4)$ & 1 & \\
\hline Missed clinic appointm & & & & & \\
\hline Never & $46(25)$ & $31(67.4)$ & 15 (32.6) & $5.5(2.5,12.4)$ & $0.000 *$ \\
\hline Ever & $74(75)$ & $20(27.0)$ & $54(73.0)$ & 1 & \\
\hline
\end{tabular}

*Significant $p<0.05$-y Pearson chi-squared test. Co-morbidities are any other diseases other than hypertension

Adherence rates varied among the PHC facilities with half of the clinics having a mean adherence $\geq 80 \%$ threshold.
There was an association between the PHC clinic attended and adherence rates ( $p=0.109)$ (Table 1; Fig. 3). 
Table 2 Validity of the Namibian version of Hill-Bone compliance scale

\begin{tabular}{|c|c|c|c|c|}
\hline \multirow{2}{*}{$\begin{array}{l}\text { Item } \\
\text { How often? }\end{array}$} & \multirow[t]{2}{*}{ Mean score $( \pm \mathrm{SD})$} & \multicolumn{3}{|c|}{ Sub-scales (principal component analysis) } \\
\hline & & $\begin{array}{l}\text { Factor } 1 \\
\text { (medication } \\
\text { taking) }\end{array}$ & $\begin{array}{l}\text { Factor } 2 \\
\text { (salt taking) }\end{array}$ & $\begin{array}{l}\text { Factor } 3 \\
\text { (appointment } \\
\text { keeping) }\end{array}$ \\
\hline Item 1: do you forget to take your hypertension medicine & $1.5 \pm 0.6$ & 0.511 & & \\
\hline $\begin{array}{l}\text { Item 2: do you decide not to take your hypertension } \\
\text { medicine }\end{array}$ & $1.2 \pm 0.4$ & 0.473 & & \\
\hline Item 3: do you eat salty food & $2.2 \pm 0.8$ & & -0.934 & \\
\hline Item 4: do you put salt on your food before you eat it & $2.3 \pm 0.8$ & & -0.935 & \\
\hline $\begin{array}{l}\text { Item 5: do you eat fast food (Kentucky Fried Chicken (KFC), } \\
\text { fat cook, fish and chips) }\end{array}$ & $2.1 \pm 0.8$ & & -0.737 & \\
\hline Item 7: do you miss scheduled appointments & $1.8 \pm 0.6$ & 0.523 & & \\
\hline $\begin{array}{l}\text { Item 8: do you leave the dispensary before } \\
\text { obtaining your prescribed pills }\end{array}$ & $1.6 \pm 0.5$ & 0.513 & & \\
\hline Item 9: do you run out of hypertension pills & $1.4 \pm 0.5$ & 0.566 & & \\
\hline $\begin{array}{l}\text { Item 10: you skip your hypertension pills } 1 \text { to } 3 \\
\text { days before you go to the clinic }\end{array}$ & $1.2 \pm 0.4$ & 0.712 & & \\
\hline $\begin{array}{l}\text { Item 11: do you miss taking your hypertension pills } \\
\text { when you feel better }\end{array}$ & $1.1 \pm 0.3$ & 0.584 & & 0.425 \\
\hline Item 13: do you take someone else's hypertension pills & $1.1 \pm 0.3$ & & & -0.757 \\
\hline $\begin{array}{l}\text { Item 14: do you miss taking your hypertension } \\
\text { pills when you care less }\end{array}$ & $1.0 \pm 0.1$ & & & -0.716 \\
\hline Overall mean score & $1.7 \pm 0.2$ & & & \\
\hline Percentage of explained variance & & 24.1 & 16.7 & 10.8 \\
\hline
\end{tabular}

Fig. 2 Adherence to antihypertensive therapy by HillBone compliance scale

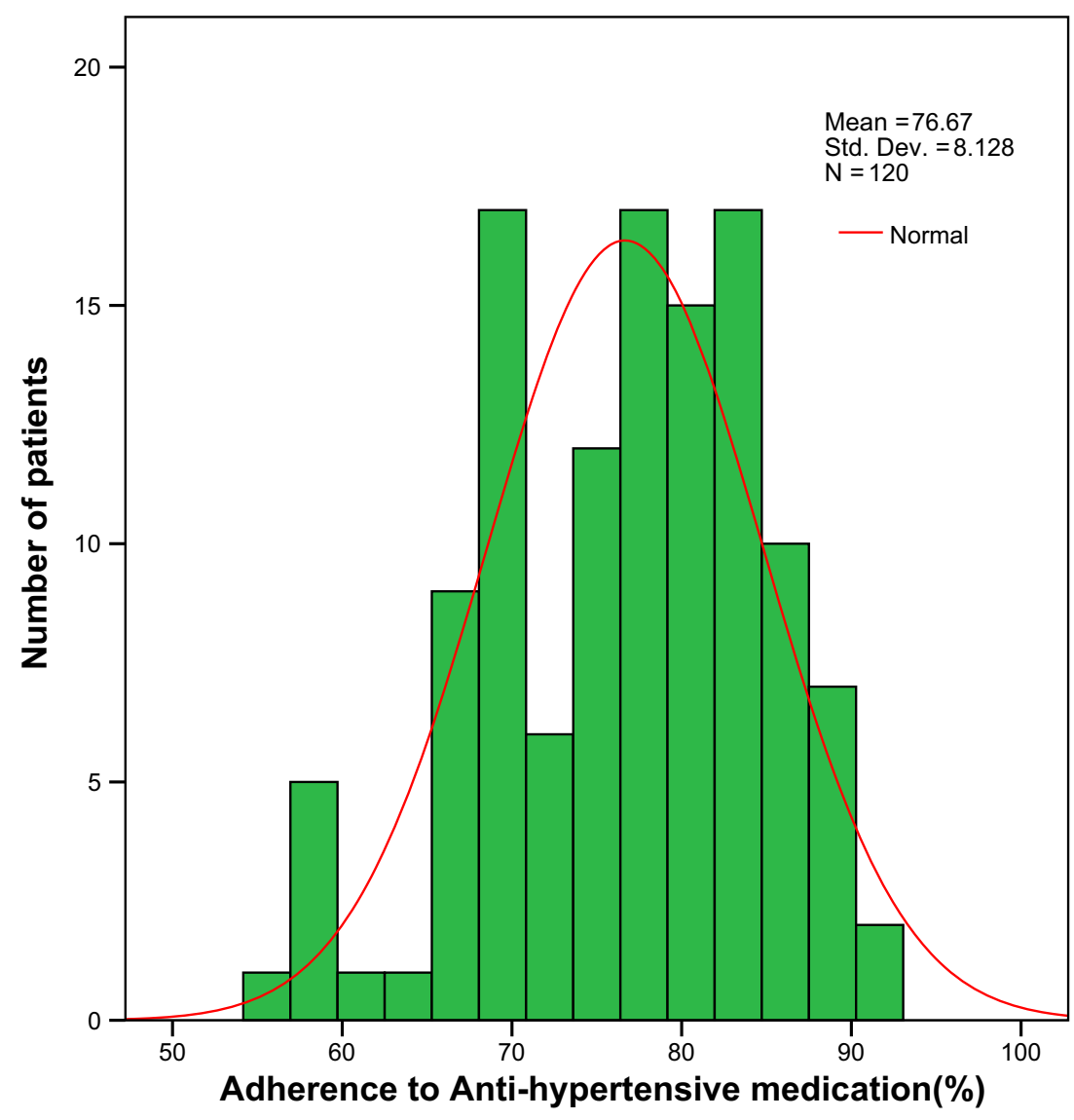


Fig. 3 Adherence to antihypertensive therapy by primary health care facility

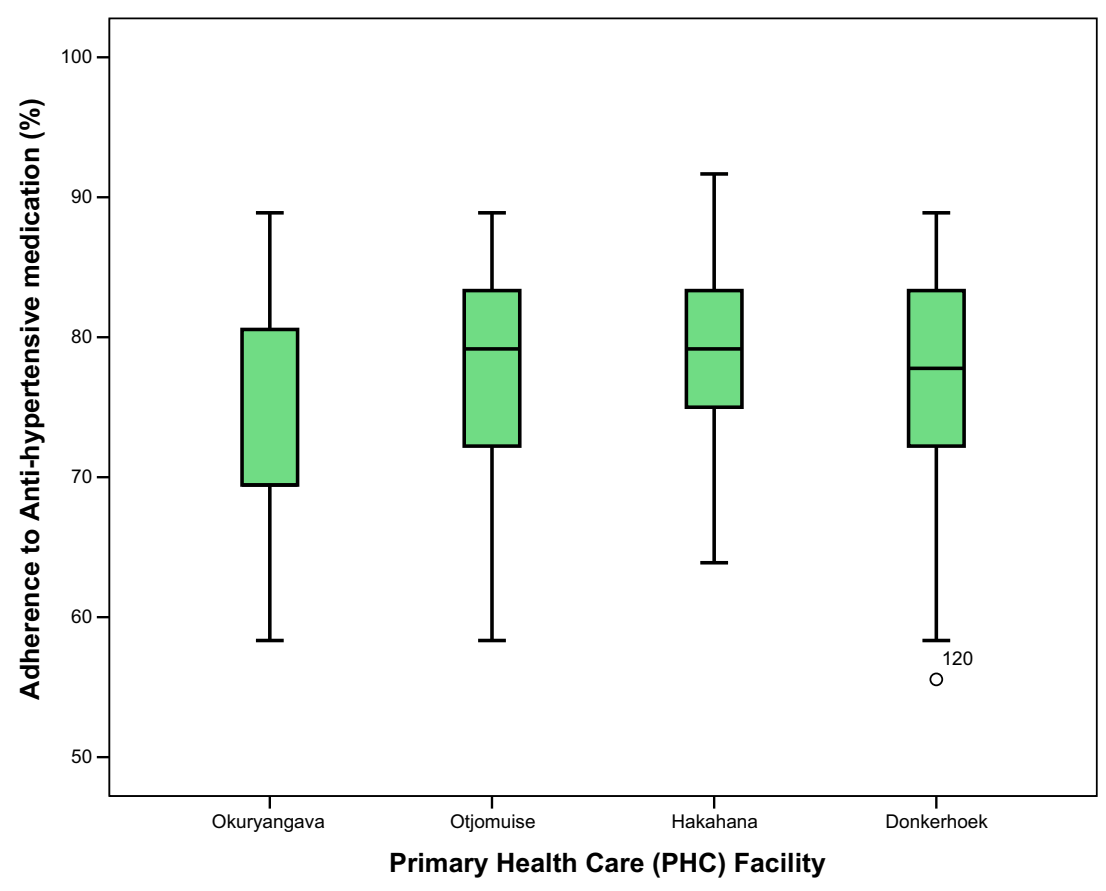

\section{Multivariate Logistic Model for Adherence to Antihypertensive Therapy}

A logistic regression analysis was conducted to identify factors associated with adherence to antihypertensive therapy (adherence $>80 \%$ ) (Table 3 ). A test of the full model against a constant only model was statistically significant, indicating that the predictors as a set reliably distinguished between adherence $(\geq 80 \%)$ and non-adherence to antihypertensive therapy $\left(\chi^{2}=36.16, p=0.001\right.$ with $\left.d f=15\right)$.

Nagelkerke's $R^{2}$ of 0.35 indicated a relationship between prediction and grouping by adherence. Prediction success overall was $71.7 \%$ (60.8\% for adherence and $79.7 \%$ for non-adherence). The Wald criterion demonstrated that only attendance of follow-up visits, having a treatment buddy, and missing clinic appointments made a significant contribution to prediction.

In the multivariate model, patient's age was not a significant predictor for adherence. Several co-variates were identified as independent predictors for adherence to antihypertensive therapy (Table 3 ). Ever having missed a clinic appointment $(\mathrm{OR}=0.2,95 \%$ CI $0.1,0.6)$, having a support buddy $(\mathrm{OR}=5.4,95 \% \mathrm{CI} 1.1,27.6)$ and always attending follow-up visits (OR $=3.1,95 \%$ CI 1.1, 8.7) were significant factors for adherence to antihypertensive therapy.

\section{Discussion}

This study aimed to validate the Hill-Bone Scale for assessing adherence to antihypertensive therapy in the primary health care in semi-urban settlements of Namibia (Table 2). The modified 12-item Namibian version of the Hill-Bone compliance scale showed reasonable internal consistence and construct validity of the three sub-scales for use to assess adherence to antihypertensive medication in primary health care in Namibia (Table 2). Previous studies in PHC settings across countries including Korea, Persia, Poland, South Africa and Turkey indicate that the Hill-Bone scale is a reliable and valid tool to assess adherence to antihypertensive therapy [13, 27, 31-34]. However, the Hill-Bone scale should be validated in the urban PHC settings in Namibia before it is universally used throughout Namibia for measurement of adherence.

This study also assessed the level and factors that may affect adherence to antihypertensive therapies as well as lifestyle and other factors that may impact on achieving control of blood pressure (Tables 1 and 3). In this study, no patient had perfect adherence to antihypertensive therapy, and over half (58\%) of the patients had adherence levels less than the designated threshold of $80 \%[6,26,30]$. This is lower than studies in South Africa and Zambia [22, 35], but comparable to other countries including Kenya and Korea [13, 36-38]. Any differences may be due to the different study and culture settings, patient characteristics, as well as sub-scales of the Hill-Bone scale used to assess compliance. In addition, we are more likely to see non-adherent patients referred to hospitals for the management of complications and/or investigations, adversely affecting documented adherence rates.

The study found a positive association between visit attendance and adherence to antihypertensive therapy, similar to other studies [6, 39]. A multivariate logistic analysis indicated that having social support, regular attendance of follow-up visits and never missed a clinic appointment were significant predictors of adherence to antihypertensive medication (Table 3). 
Table 3 Multivariate logistic model for adherence to antihypertensive therapy

\begin{tabular}{|c|c|c|}
\hline Covariates & Adjusted OR (95\% CI) & $p$ value \\
\hline \multicolumn{3}{|c|}{ PHC distance from state hospital } \\
\hline$<3 \mathrm{~km}$ & 1.0 & \\
\hline$>3 \mathrm{~km}$ & $0.8(0.3,2.3)$ & 0.717 \\
\hline \multicolumn{3}{|c|}{ Patient's age (years) } \\
\hline & 1.0 & \\
\hline & $2.6(0.9,7.7)$ & 0.084 \\
\hline \multicolumn{3}{|c|}{ Patient's sex (years) } \\
\hline & 1.0 & \\
\hline & $1.2(0.5,3.2)$ & 0.703 \\
\hline \multicolumn{3}{|c|}{ Secondary education level } \\
\hline Yes & 1.0 & \\
\hline No & $0.7(0.3,2.0)$ & 0.515 \\
\hline \multicolumn{3}{|c|}{ Employment status } \\
\hline Employed & 1.0 & \\
\hline Unemployed & $1.1(0.4,2.8)$ & 0.903 \\
\hline \multicolumn{3}{|l|}{ Marital status } \\
\hline Married & 1.0 & \\
\hline Single & $1.9(0.7,4.9)$ & 0.208 \\
\hline \multicolumn{3}{|c|}{ Years with hypertension (years) } \\
\hline$<5$ years & 1.0 & \\
\hline$>5$ years & $0.6(0.2,1.6)$ & 0.332 \\
\hline \multicolumn{3}{|c|}{ Chronic co-morbidity } \\
\hline Yes & 1.0 & \\
\hline No & $1.0(0.4,2.5)$ & 0.923 \\
\hline \multicolumn{3}{|c|}{ Received adequate refill on last visit } \\
\hline Yes & 1.0 & \\
\hline No & $0.8(0.2,2.7)$ & 0.682 \\
\hline \multicolumn{3}{|c|}{ Adequately informed on use of medicines } \\
\hline Yes & 1.0 & \\
\hline No & $1.8(0.5,6.4)$ & 0.401 \\
\hline \multicolumn{3}{|c|}{ Knows consequences of hypertension } \\
\hline No & 1.0 & \\
\hline Yes & $0.7(0.3,2.0)$ & 0.534 \\
\hline \multicolumn{3}{|c|}{ Always attends follow-up visits } \\
\hline No & 1.0 & \\
\hline Yes & $3.1(1.1,8.7)$ & $0.030 *$ \\
\hline \multicolumn{3}{|c|}{ Has support buddy } \\
\hline No & 1.0 & \\
\hline Yes & $5.4(1.1,27.6)$ & $0.045^{*}$ \\
\hline \multicolumn{3}{|c|}{ Missed a clinic appointment } \\
\hline Never & 1.0 & \\
\hline Ever & $0.2(0.1,0.6)$ & $0.002 *$ \\
\hline Constant & 0.5 & 0.613 \\
\hline
\end{tabular}

Variable(s) entered on step 1: treatment support buddy, always attends visits, age of patient, ever missed clinic appointment

Similar studies have reported the lack of treatment support buddies and/or a spouse as an important risk factor for non- adherence to antihypertensive medication, particularly among the elderly $[6,37,40]$. There will be further ongoing researchinvestigating issues such as social support in more depth given its importance in helping to improve future adherence rates.

Despite the fact that most patients often forget to make a suitable appointment date for the next clinic (Hill-Bone score mean 3.9 out of 4), missing of appointments was common (Table 2). In this study, the majority of patients missed at least one or more of their follow-up visit. The main reasons for missing follow-up visits were the lack of transport to the facility, forgetting the appointment dates, work-related pressures and feeling unwell.

The discrepancy between knowing the date for the next visit (appointment date) and actually turning up for an appointment may require added benefits or incentives for attending and/or the availability of a system to track the patients. There may also be a need for a reminder system for clinic appointments and refill appointments in Namibia to enhance adherence to visits and treatment. In addition, critically looking at issues such as transport, where this is a concern, as well as flexibility of opening hours of the PHCs given the high prevalence of hypertension in Namibia and the fact that there is currently no co-payment for these medicines. Longer distance also negatively impacted on adherence rates in a study in Northwest Ethiopia, especially when it was accompanied by poor infrastructure [41]. Extending available personnel and systems to manage these patients could help, which could include additional nurses and pharmacists [42, 43].

In this study, despite approximately eight out of ten patients having a literacy level of primary education and above, adherence to antihypertensive therapy was still sub-optimal. This is in contrast with previous studies in Africa and elsewhere that have associated non-adherence to antihypertensive therapy to lowliteracy levels defined by Saounatsou as years of schooling and Yiannakopoulou et al. as below lyceum or university $[6,44,45]$. This discrepancy may be in part explained by the fact that less than half of the patients in this study were literate about the consequences of uncontrolled high blood pressure (Table 1). However, we did not find any significant association between adherence and literacy on antihypertensive medication $(p=0.594)$. The non-adherence to antihypertensive therapy in this study may also though be due in part to the low socioeconomic status of the study population, which may negatively impact on health care seeking behaviours especially if there are transport difficulties [41]. The limited capacity for monitoring adherence to antihypertensive therapy at the PHC clinics in Namibia may also negatively impact on adherence despite universal access.

Our findings are different from those of other studies that have associated adherence to the level of education, complications of hypertension, antihypertensive dosage regimen, concomitant chronic disease states, patients' age, access to medicines, quality of care and attendance of follow-up visits [6, 39, 46-49]. These 
differences may be due to the fact that our study was conducted among a homogenous population - among people of a low socioeconomic status and at primary level of care. Two thirds of the respondents in this study were from one ethnic group, Oshiwambo, and adherence may be influenced by the local culture. A number of studies have been conducted at the hospital level, which usually have more diverse populations of respondents and prescribers. A homogenous population is more likely to have similar behaviours compared to a heterogeneous one. We plan to confirm this in future studies.

There was also no significant association between adherence and patients' demographic characteristics including patient's age, sex, employment status, education level as well as literacy on hypertension therapy (Table 3) and having another chronic co-morbidity such as HIV/AIDS or diabetes alongside hypertension. No association with concomitant chronic illness such as HIV/AIDS is an interesting finding, especially as concomitant HIV/AIDs will appreciably increase the pill burden, which is known to adversely affect adherence rates $[48,50]$. This may be because HIV/AIDS patients are more regularly monitored and counselled about medication adherence, which itself may influence adherence rates across disease areas despite appreciably increasing the number of pills taken each day. We have seen this in other NCD disease areas in Africa such as diabetes whereby adherence rates may in fact be increased if patients have concomitant HIV. This may be because these co-morbid patients feel better cared for than those with only hypertension, positively impacting on adherence rates in practice [51]. We plan to follow this up in future research studies as the rationale will provide additional guidance on ways to further improve adherence to antihypertensive medicines in Namibia.

\section{Limitations}

We accept this study has a number of limitations. The principal one includes the limited sample of hospitals included (four clinics in the informal settlements of Windhoek, the capital city of Namibia) and the non-inclusion of the three clinics in the central business district of Windhoek. We are currently conducting a similar study among the remaining PHC facilities in central Windhoek and aim to compare the findings from these two studies. Nevertheless, we believe that the findings of this study alone are important in providing evidence which can guide efforts to improve adherence to antihypertensive therapy among patients of low social economic status in Namibia.

\section{Conclusions}

The modified 12-item Namibian version of the Hill-Bone compliance scale is a reliable and valid tool for assessing adherence to antihypertensive therapy in semi-urban settings of Namibia.

There is currently sub-optimal adherence to antihypertensive therapy among patients attending the PHC facilities, which is a concern. Irregular attendance of follow-up visits, lack of treatment support and missing appointments are important risk factors for adherence to antihypertensive medication in our study. Distance was also important in the bivariate but not multivariate analysis. As a result, there is need for standard packages in antihypertensive therapy at PHCs, as well as a system to monitor and remind patients of their follow up-visits, to address current concerns. This could involve mHealth techniques and mobile reminder systems in the future. There is also a need to build capacity to initiate and monitor antihypertensive therapy at the PHC level. This may mean making PHCs becoming more flexible when they can see patients as well as looking at using other professionals in care delivery such as community pharmacists.

Incentives could potentially be offered to patients to address identified barriers. Assessing the rationale behind similar medication adherence rates between hypertensive patients with and without HIV/AIDs is also likely to help with programmes to improve future adherence rates. Alongside, this is assessing the actual impact of these activities on improving long-term blood pressure control.

The outcomes of this study will inform the development of appropriate strategies in PHCs in Namibia, integrating treatment literacy services, treatments and outcomes at all or specific points of care. Potential target areas include counseling, prescribing, dispensing and follow-up of patients. Ultimately, patients should be empowered to monitor their own clinical outcomes and adherence to antihypertensive therapy. In addition, investigating further issues such as transport and what can be learnt from patients jointly having hypertension and HIV, given the appreciable burden of both in Sub-Saharan countries. Once underway, seek to potentially instigate screening programmes to reduce the morbidity, mortality and costs associated with hypertension in Namibia given the likely extent of undiagnosed hypertension. This will help Namibia achieve SDG 3.4.

\section{Compliance with Ethical Standards}

Ethics The study was approved by the research and ethics committee of the Faculty of Health Sciences, UNAM and the Ministry of Health and Social Services (MoHSS). All patients who participated in the study gave written informed consent. The management of the four PHCs, i.e. Okuryangava HC, Otjomuise, Donkerhoek and Hakahana, approved the study. In order to maintain confidentiality, questionnaires were coded and no patient specific identifiers such as names and hospital numbers were collected.

Informed Consent All patients who participated in the study gave written informed consent.

Conflict of Interest The authors declare that they have no conflicts of interest. 


\section{Appendix A}

\section{QUESTIONNAIRE}

\begin{tabular}{l|l|}
\hline & No: \\
\cline { 1 - 1 } QUESTIONAIRE & \\
\hline
\end{tabular}

Date:

Hello. I would like to thank you for taking time to complete this questionnaire. This is to help me with my university research on Medication Adherence among Hypertensive Patients attending Four Clinics in Windhoek for Follow-ups. I therefore implore you to please answer truthfully so that the results of the research will be of sound quality. All the information will be kept confidential. If you have any questions while filling in this questionnaire, please ask the questionnaire administrator. Thank you.

\section{Please $(\sqrt{ })$ where appropriate}

Section A: DEMOGRAPHIC DATA

1. Age (at last birthday)

2. Sex

Male

3. Highest level of Education reached:

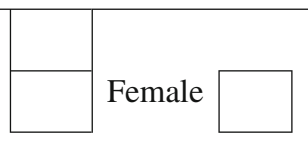

a. Tertiary Education

b. Secondary Education (grade 8-12)
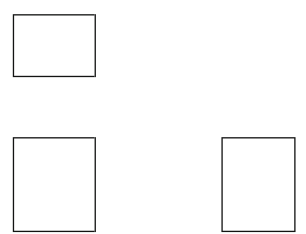

c. Primary Education (grade 1-7)
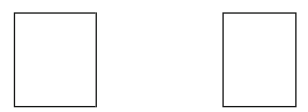

d. None

4. Occupation

a. Student
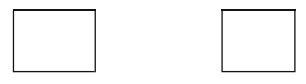

b. Employed
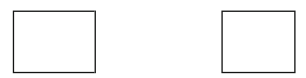

c. Self-employed

d. Unemployed

5.Marital status

Single

Married

In a relationship

6.Ethnicity 
SECTION B: Personal Medical History

1. When were you diagnosed with High blood pressure? (year)

2. Do you have any other chronic illness(es)

3. If yes, please mention it (them)

4. At your last visit at the clinic for your high blood pressure follow-up, did you receive enough medication to last you until your next visit?

5. Did your nurse or doctor tell you when, how and why you need to take your high blood pressure medication every time you are supposed to?

6. Do you know what might happen if you do not take your medication every time you are supposed to?

7. Do your friends and family encourage you to take your medication?

8. Do your friends and family accompany you to the clinic for follow-ups?

9. Do you always go to your follow-ups on the days you are supposed to?

10. If not, please say why? - 
SECTION C: Hill-Bone Compliance to High Blood Pressure Therapy Scale

(Please answer the following questions in the table below, by ticking appropriately.)

1. How often do you forget to take your hypertension medicine?
None of the time
Some of the time
Most of the time
All the time

2. How often do you decide not to take your hypertension medicine?

None of the time $\square \quad$ Some of the time $\square \quad$ Most of the time $\square \quad$ All the time

3. How often do you eat salty food?

None of the time $\square \quad$ Some of the time $\square \quad$ Most of the time $\square \quad$ All the time

4. How often do you put salt, Fondor or Aromat on your food before you eat it?
None of the time
Some of the time
Most of the time
All the time

5. How often do you eat fast food (KFC, kapana, fat cook, fish and chips)?

None of the time $\square \quad$ Some of the time $\square \quad$ Most of the time $\square$ All the time

6. How often do you forget to make the next clinic appointment before you leave the clinic?

None of the time $\square \quad$ Some of the time $\square \quad$ Most of the time $\square$ All the time

7. How often do you miss scheduled appointments?

None of the time $\square \quad$ Some of the time $\square \quad$ Most of the time $\square \quad$ All the time

8. How often do you leave the dispensary before obtaining your prescribed pills (due to long line, closure of clinic, forgetting)?
None of the time
Some of the time
Most of the time
All the time

9. How often do you run out of hypertension pills?
None of the time
Some of the time
Most of the time
All the time

10. How often do you skip your hypertension pills 1 to 3 days before you go to the clinic?
None of the time
Some of the time
Most of the time
All the time

11. How often do you miss taking your hypertension pills when you feel better?
None of the time
Some of the time
Most of the time
All the time

12. How often do you miss taking your hypertension pills when you feel sick?
None of the time
Some of the time
Most of the time
All the time

13. How often do you take someone else's hypertension pills?

None of the time

Some of the time

Most of the time

All the time

14. How often do you miss taking your hypertension pills when you care less?

None of the time

Some of the time

Most of the time

All the time 
Open Access This article is distributed under the terms of the Creative Commons Attribution 4.0 International License (http:// creativecommons.org/licenses/by/4.0/), which permits unrestricted use, distribution, and reproduction in any medium, provided you give appropriate credit to the original author(s) and the source, provide a link to the Creative Commons license, and indicate if changes were made.

\section{References}

1. World Health Organisation (WHO). A global brief on hypertension - silent killer, global public health crisis. World Health Day 2013. Available at URL: http://apps.who.int/iris/bitstream/10665/ 79059/1/WHO_DCO_WHD_2013.2_eng.pdf?ua=1

2. Ataklte F, Erqou S, Kaptoge S, Taye B, Echouffo-Tcheugui JB, Kengne AP. Burden of undiagnosed hypertension in Sub-Saharan Africa: a systematic review and meta-analysis. Hypertension. 2015;65(2):291-8.

3. Lawes CM, Vander Hoorn S, Rodgers A. Global burden of bloodpressure-related disease, 2001. Lancet. 2008;371(9623):1513-8.

4. Irazola VE, Gutierrez L, Bloomfield G, Carrillo-Larco RM, Dorairaj P, Gaziano T, et al. Hypertension prevalence, awareness, treatment, and control in selected low and middle income countries (LMIC) communities: results from the NHLBI/UHG Network of Centers of Excellence for Chronic Diseases. Glob Heart. 2016;11(1):47-59.

5. World Health Organisation, Global Health Observatory (GHO) data. Raised blood pressure. Available at URL: http://www.who.int/ gho/ncd/risk factors/blood pressure prevalence text/en/

6. Nielsen JO, Shrestha AD, Neupane D, Kallestrup P. Non-adherence to anti-hypertensive medication in low- and middle-income countries: a systematic review and meta-analysis of 92443 subjects. J Hum Hypertens. 2017;31(1):14-21.

7. Hendriks ME, Wit FWNM, Roos MTL, Brewster LM, Akande TM, de Beer IH, et al. Hypertension in Sub-Saharan Africa: cross-sectional surveys in four rural and urban communities. PLoS One. 2012;7(3):e32638.

8. Chobanian AV, Bakris GL, Black HR, Cushman WC, Green LA, Izzo JL Jr, et al. Seventh report of the Joint National Committee on prevention, detection, evaluation, and treatment of high blood pressure. Hypertension. 2003;42(6):1206-52.

9. World Health Organisation. Namibia: World Health Organisation statistical profile. Available at URL: http://www.who.int/gho/ countries/nam.pdf?ua=1

10. The Nambia Ministry of Health and Social Services (MoHSS) and ICF International. 2014. The Namibia Demographic and Health Survey 2013. Windhoek, Namibia, and Rockville, Maryland, USA: MoHSS and ICF International. Available at URL: https:// dhsprogram.com/pubs/pdf/FR298/FR298.pdf.

11. Corrao G, Parodi A, Nicotra F, Zambon A, Merlino L, Cesana G, et al. Better compliance to antihypertensive medications reduces cardiovascular risk. J Hypertens. 2011;29(3):610-8.

12. World Health Organisation. Millennium developmet goals sustainable development goals Chapter 6. Noncommunicable diseases. Available at URL: http://www.who.int/gho/publications/mdgssdgs/MDGs-SDGs2015 chapter6.pdf?ua=1

13. Krousel-Wood M, Thomas S, Muntner P, Morisky D. Medication adherence: a key factor in achieving blood pressure control and good clinical outcomes in hypertensive patients. Curr Opin Cardiol. 2004;19(4):357-62.

14. Calhoun DA, Jones D, Textor S, Goff DC, Murphy TP, Toto RD, et al. Resistant hypertension: diagnosis, evaluation, and treatment. A scientific statement from the American Heart Association
Professional Education Committee of the Council for High Blood Pressure Research. Hypertension. 2008;51(6):1403-19.

15. Mant J, McManus RJ. Does it matter whether patients take their antihypertensive medication as prescribed? The complex relationship between adherence and blood pressure control. J Hum Hypertens. 2006;20(8):551-3.

16. Ministry of Health and Social Services. Namibia essential medicines list [Nemlist] 5th edition. Republic of Namibia Ministry of Health and Social Services, Windhoek, Namibia. Available at URL: http:// apps.who.int/medicinedocs/documents/s23017en/s23017en.pdf

17. Ministry of Health and Social Services. Namibia Standard treatment guidelines 1st edition. Republic of Namibia Ministry of Health and Social Services Windhoek Namibia. Available at URL: http://apps. who.int/medicinedocs/documents/s19260en/s19260en.pdf

18. Ministry of Health and Social Services. National medicines policy 2nd edition. Republic of Namibia Ministry of Health and Social Services, Windhoek, Namibia. 2011.

19. Ministry of Health and Social Services. Republic of Namibia. Medicines and related substances control Act, 13, Republic of Namibia, 2003. Available at URL: http://www.wipo.int/edocs/ lexdocs/laws/en/na/na017en.pdf

20. Cameron A, Ewen M, Ross-Degnan D, Ball D, Laing R. Medicine prices, availability, and affordability in 36 developing and middleincome countries: a secondary analysis. Lancet. 2009;373(9659): $240-9$.

21. Bell R, Ithindi T, Low A. Improving equity in the provision of primary health care: lessons from decentralized planning and management in Namibia. Bull World Health Organ. 2002;80(8):675-81.

22. Mweene D, Banda J, Andrews B, M-Mweene M, Lakhi S. Factors associated with poor medication adherence in hypertensive patients in Lusaka, Zambia. Med J Zambia. 2010;37(3):252-61.

23. WHO. How to investigate drug use in health facilities: selected drug use Indicators-EDM Research Series No. 007. Available at URL: http://apps.who.int/medicinedocs/en/d/Js2289e/

24. Kim MT, Hill MN, Bone LR, Levine DM. Development and testing of the hill-bone compliance to high blood pressure therapy scale. Prog Cardiovasc Nurs. 2000;15(3):90-6.

25. Sarker SK, Bt Kamal NAI, Bin Rohazaki AA, Rashid AFBZ, Bt Mansor F, Halim NBA, et al. Prevalence of patients compliance among hypertensive patients and its associated factors in Klinik Kesihatan Botanic Klang, Malaysia. Int J Intg Med Sci. 2016;3(7):345-9.

26. Dehghan M, Nayeri ND, Iranmanesh S. Validating the Persian version of the Hill-Bone's scale of "compliance to high blood pressure therapy". Br J Med Med Res. 2015;5(2):235-46.

27. Lambert EV, Steyn K, Stender S, Everage N, Fourie JM, Hill M. Cross-cultural validation of the hill-bone compliance to high blood pressure therapy scale in a South African, primary healthcare setting. Ethn Dis. 2006;16:286-91.

28. Culig J, Leppee M. From Morisky to Hill-bone; self-reports scales for measuring adherence to medication. Coll Antropol. 2014;38(1): 55-62.

29. Berra E, Azizi M, Capron A, Hoieggen A, Rabbia F, Kjeldsen SE, et al. Evaluation of adherence should become an integral part of assessment of patients with apparently treatment-resistant hypertension. Hypertension. 2016;68(2):297-306.

30. Inkster ME, Donnan PT, MacDonald TM, Sullivan FM, Fahey T. Adherence to antihypertensive medication and association with patient and practice factors. J Hum Hypertens. 2006;20(4):295-7.

31. Karademir M, Koseoglu IH, Vatansever K, Van Den Akker M. Validity and reliability of the Turkish version of the Hill-Bone compliance to high blood pressure therapy scale for use in primary health care settings. Eur J Gen Pract. 2009;15(4):207-11.

32. Lavsa SM, Holzworth A, Ansani NT. Selection of a validated scale for measuring medication adherence. J Am Pharm Assoc. 2011;51(1):90-4. 
33. Song Y, Han HR, Song HJ, Nam S, Nguyen T, Kim MT. Psychometric evaluation of hill-bone medication adherence subscale. Asian Nurs Res. 2011;5(3):183-8.

34. Uchmanowicz I, Jankowska-Polańska B, Chudiak A, SzymańskaChabowska A, Mazur G. Psychometric evaluation of the polish adaptation of the hill-bone compliance to high blood pressure therapy scale. BMC Cardiovasc Disord. 2016;16:87.

35. Mafutha GN, Wright SC. Compliance or non-compliance of hypertensive adults to hypertension management at three primary healthcare day clinics in Tshwane. Curationis. 2013;36(1):E1-6.

36. Acheing L, Joshi M, Ogola EN, Karari E. Adequacy of blood pressure control and level of adherence with antihypertensive therapy. East Afr Med J. 2009;86(11):499-506.

37. Demoner MS, Ramos ERDP, Pereira ER. Factors associated with adherence to antihypertensive treatment in a primary care unit. Acta Paulista de Enfermagem. 2012;25(Special Issue 1):27-34.

38. Awad EY, Gwaied BE, Fouda LM, Essa HAE-GE. Compliance of hypertensive patients with treatment regimen and its effect on their quality of life. J Nurs Health Sci. 2015;4(2):26-36.

39. Jones JK, Gorkin L, Lian JF, Staffa JA, Fletcher AP. Discontinuation of and changes in treatment after start of new courses of antihypertensive drugs: a study of a United Kingdom population. BMJ. 1995;311(7000):293-5.

40. Ma C. A cross-sectional survey of medication adherence and associated factors for rural patients with hypertension. Appl Nurs Res. 2016;31:94-9.

41. Ambaw AD, Alemie GA, W/Yohannes SM, Mengesha ZB. Adherence to antihypertensive treatment and associated factors among patients on follow up at University of Gondar Hospital, Northwest Ethiopia. BMC Public Health. 2012;12:282.

42. Coleman R, Gill G, Wilkinson D. Noncommunicable disease management in resource-poor settings: a primary care model from rural South Africa. Bull World Health Organ. 1998;76(6):633-40.
43. Erhun WO, Agbani EO, Bolaji EE. Positive benefits of a pharmacist-managed hypertension clinic in Nigeria. Public Health. 2005;119(9):792-8.

44. Saounatsou M, Patsi O, Fasoi G, Stylianou M, Kavga A, Economou O, et al. The influence of the hypertensive patient's education in compliance with their medication. Public Health Nurs. 2001;18(6):436-42.

45. Yiannakopoulou E, Papadopulos JS, Cokkinos DV, Mountokalakis TD. Adherence to antihypertensive treatment: a critical factor for blood pressure control. Eur J Cardiovasc Prev Rehabil. 2005;12(3):243-9.

46. Mugwano I, Kaddumukasa M, Mugenyi L, Kayima J, Ddumba E, Sajatovic M, et al. Poor drug adherence and lack of awareness of hypertension among hypertensive stroke patients in Kampala, Uganda: a cross sectional study. BMC research notes. 2016;9:3.

47. Adams AS, Uratsu C, Dyer W, Magid D, O'Connor P, Beck A, et al. Health system factors and antihypertensive adherence in a diverse cohort of new users. JAMA Intern Med. 2013;173(1):54-61.

48. Akeroyd JM, Chan WJ, Kamal AK, Palaniappan L, Virani SS. Adherence to cardiovascular medications in the South Asian population: a systematic review of current evidence and future directions. World J Cardiol. 2015;7(12):938-47.

49. Harmon G, Lefante J, Krousel-Wood M. Overcoming barriers: the role of providers in improving patient adherence to antihypertensive medications. Curr Opin Cardiol. 2006;21(4):310-5.

50. Mancia G, Fagard R, Narkiewicz K, Redon J, Zanchetti A, Bohm M, et al. 2013 European Society of Hypertension/European Society of Cardiology(ESH/ESC) guidelines for the management of arterial hypertension: the task force for the management of arterial hypertension. J Hypertens. 2013;31(7):1281-357.

51. Rwegerera G, Moshomo T, Gaenamong M, Oyewo TA, Gollakota S, Mhimbira FA, et al. Antidiabetic medication adherence and associated factors among patients in Botswana; implications for the future. Alex J Med. 2017; https://doi.org/10.1016/j.ajme.2017.01.005. 